\title{
Dynamic Interference Optimization in Cognitive Radio Networks for Rural and Suburban Areas
}

\author{
Rodney Martinez Alonso $\mathbb{D}^{1},{ }^{1,2}$ David Plets $\mathbb{D}^{\mathbb{1}},{ }^{1}$ Margot Deruyck $\mathbb{D}^{1},{ }^{1}$ Luc Martens $\mathbb{D}^{\mathbb{D}}{ }^{1}$ \\ Glauco Guillen Nieto ${ }^{\mathbb{D}},{ }^{2}$ and Wout Joseph ${ }^{1}$ \\ ${ }^{1}$ Information Technology, Ghent University, Gent 9052, Belgium \\ ${ }^{2}$ R\&D Telecom, LACETEL, Havana 19200, Cuba
}

Correspondence should be addressed to Rodney Martinez Alonso; rodney.martinezalonso@ugent.be

Received 21 November 2019; Accepted 27 April 2020; Published 9 May 2020

Academic Editor: Luciano L. Mendes

Copyright (c) 2020 Rodney Martinez Alonso et al. This is an open access article distributed under the Creative Commons Attribution License, which permits unrestricted use, distribution, and reproduction in any medium, provided the original work is properly cited.

In this paper, we investigate the coexistence of cognitive radio networks on TV white spaces for rural and suburban connectivity. Although experimental models and laboratory measurements defined the maximum interference threshold for TV white space technologies for general use cases, our research found that in real wireless rural and suburban scenarios, severe interference to the broadcasting services might occur. This is particularly relevant when the traffic load of the telecom base stations (BSs) exceeds $80 \%$ of their maximum capacity. We propose a dynamic management algorithm for minimizing the interference, based on a centralized access control architecture for cognitive radio wireless networks. In an experimental emulation for assessing the impact of cognitive radio interference on the broadcasting service's QoE, our method reduced the perceived video distortion by the broadcasting users by at least $50 \%$ and $27.5 \%$ in a rural and suburban scenario, respectively, while the spectrum usage is increased by just $8 \%$.

\section{Introduction}

The development of more efficient modulation schemes and forward error correction codes has allowed reducing the gap to the theoretical Shannon capacity limit. For instance, the introduction of LDPC (Low-Density Parity Check) codes allowed the last generation of broadcasting television technologies to get within $2 \mathrm{~dB}$ of the theoretical capacity limit (Shannon limit) [1]. The modulation and coding schemes (MCS) developed for ATSC 3.0 digital television standard are $1.3 \mathrm{~dB}$ to $1.8 \mathrm{~dB}$ from the Shannon limit [2]. This improvement leads to a higher broadcasting spectral efficiency. This allowed releasing hundreds of megahertz for wireless and mobile communications services, i.e., the $700 \mathrm{MHz}$ band has been reassigned in several countries for mobile communications $[3,4]$. Despite the increasing improvements in the spectral efficiency of technologies, the demand for bandwidth exceeds the availability of spectrum for new communication services and networks. Paradoxically, several spectrum sur- veys demonstrate that the spatial and temporal use of the sub $3 \mathrm{GHz}$ spectrum is less than $20 \%[5,6,7,8]$. This is a direct consequence of the inefficient allocation and assignment of the spectrum. Hence, nowadays, the main problem is not related to technology efficiency itself but how to use it efficiently.

Cognitive radio paradigms over different regulatory constraints have been proposed as a long-term solution for tackling spectrum scarcity for broadband wireless services and the lack of spectrum usage efficiency. Different standards enabling cognitive radio technologies for spectrum allocation have emerged in recent years, e.g., IEEE 802.11af [9], IEEE 802.22b [10], and ECMA 392 [11]. The importance of cognitive radio technologies and dynamic spectrum access for enabling next-generation wireless networks has also been recognized by the European Telecommunications Standards Institute (ETSI) and the Third Generation Partnership Project (3GPP) [12]. ETSI technical report TR 103 067/2013 [13] analyzes the feasibility of Long-Term Evolution (LTE) 
Cognitive Radio Systems operating in UHF band TV white spaces (TVWS). This technical report also evaluates the coexistence between LTE networks operating on TVWS and television broadcasting services [13].

The large bandwidth requirement for $5 \mathrm{G}$ networks is only fully satisfied at the highest spectrum bands. As the cells' coverage is considerably reduced, the application cases for $5 \mathrm{G}$ networks are quite limited for small cities, rural scenarios, and in general, in areas with low population density. In [14], the authors investigated the feasibility of $5 \mathrm{G}$ network operation based on cognitive radio and carrier aggregation for enabling wideband access in rural areas. In [15], a multilayer routing strategy for enabling $5 \mathrm{G}$ services exploiting TVWS is presented. They guarantee a framework for coexistence with heterogeneous networks sharing the same spectrum bands. On the other hand, the Ericsson 5G platform will utilize dynamic spectrum sharing techniques for granting spectrum access to either $4 \mathrm{G}$ or $5 \mathrm{G}$ user devices [16].

Optimizing spectrum usage efficiency without affecting the users' Quality of Service (QoS) and Quality of Experience (QoE) is critical for the future development of wireless communications operating in TVWSs. The novelty of this paper is a dynamic interference management algorithm for reducing harmful interference to broadcasting services. The algorithm is designed for operating in a centralized spectrum management architecture, rather than the traditional distributed architecture. The designed algorithm seeks a higher spectrum usage efficiency and is validated by measurements in an emulation test with self-developed testbed hardware.

For evaluating the results achieved by our algorithm, we emulate the critical conditions in terms of interference to the broadcasting station. For the first time, we assess the degradation of the broadcasting service Quality of Experience caused by secondary cognitive radio interference. We also compare the interference in a traditional cognitive radio network and the proposed optimized networks. In this research, we consider a Long-Term Evolution-Advanced (LTE-A) network operating on TVWS under the ETSI regulatory conditions for a rural scenario and suburban scenario in Havana, Cuba, and Ghent, Belgium, respectively.

The outline of this paper is as follows. In Section 2, we present the most relevant state-of-the-art related to cognitive radio technologies, in Section 3 we present the method for the dual-objective dynamic network optimization and for the experimental evaluation of the QoE in the broadcasting networks for different interference profiles generated by the optimization software. In Section 4, we present the cognitive LTE-A network optimization results and perceived degradation reduction for the broadcasting users in terms of QoE. In Section 5, the conclusions of our research are formulated.

\section{State of the Art}

Interference to and from the primary licensed service has been a major concern related to cognitive radio technologies, although sensing techniques for allocating empty channels have been improved throughout recent years [17, 18, 19].
2.1. Protection Limits. The protection limits, cochannel, and adjacent channel interference between cognitive radio and digital television technologies have been widely investigated. In [20], the authors studied the coexistence between the IEEE 802.22 cognitive radio standard and the DVB-T2 digital broadcasting. The IEEE 802.22 standard itself defined the maximum signal level threshold of ATSC for reusing the spectrum by secondary services without causing harmful interference to the primary ATSC service [21]. However, the IEEE standards (latest update) do not provide protection ratios for other digital television broadcasting services [10]. Further, the FCC and ETSI regulation defined the maximum radiated signal level for minimizing the interference among primary and secondary services $[13,22]$.

Laboratory measurements for assessing the protection of DVB-T2 broadcasting services interfered by IEEE 802.22 Wireless Regional Area Network (WRAN) transmissions in the cochannel and adjacent channels were performed, and protection ratios were defined [20]. Further research is required for studying the maximum device density and distribution [20]. For unspecified transmissions in the IEEE 802.22 standard, the suggested protection limit is $-90 \mathrm{dBm}$. However, this value is quite high for most propagation environments and broadcasting transmitters for Digital Terrestrial Multimedia Broadcast (DTMB) and Digital Video BroadcastingTerrestrial (DVB-T/T2). For the average DVB-T transmitter configuration, an additional extension of the protected broadcasting transmitter area by 5 to $15 \mathrm{~km}$ is recommended. The extended protection is estimated based on the link budget for the digital broadcasting transmitters and the interference protection margin for DVB-T2 and DTMB presented in [20] and [23], respectively. A more appropriate spectrum reusage threshold can be set at $-95 \mathrm{dBm}$ for both DVBT2 and DTMB use cases. Nevertheless, these values do not guarantee that the channel is useful for providing the desired network QoS and QoE for the secondary wireless communication service. For the primary licensed service, the protection margin should be analyzed on a case-by-case basis as the transmitters' setups and propagation conditions significantly vary.

For one of the highest modulations and coding schemes for IEEE 802.22 (64-QAM FEC=3/4), a minimum Signalto-Noise-Ratio (SNR) ratio of $19.7 \mathrm{~dB}$ is required for achieving at least $95 \%$ of the maximum throughput. The sensitivity of commercial receivers for this MCS is $-82.5 \mathrm{dBm}$. At the broadcasting edge of the protected contour with a mean signal level of $-95 \mathrm{dBm}$, the SNR is $12.5 \mathrm{~dB}$. According to the experimental results presented in [24], this increases the bit error rate beyond the maximum allowable threshold of $2 \times$ $10^{-4}$. For the worst-case coexistence scenario, being a BS located around such an area, its coverage might be reduced to approximately a third of its maximum radius $(6.8 \mathrm{~km}$ of $20 \mathrm{~km})$ [24].

The usage of these channels by the secondary wireless devices located on the border of the protected contour of the broadcasting transmitter might require dynamic management of the MCS and interference for satisfying the QoS requirements of all network users while keeping a high spectrum usage efficiency. 
2.2. Interference and Coexistence. Coexistence and interference management for TV white spaces is even more complicated due to the fact that besides the primary licensed service, several secondary services with different physical layers and medium access control mechanisms might access the spectrum in a particular area at the same time, e.g., IEEE 802.11af (either Carrier Sense Multiple Access with Collision Avoidance (CSMA/CS) or Time Division Multiplex Medium Access (TDMA)), IEEE 802.22 (TDMA with physical layer resources allocated on-demand using OFDMA) [25], and LTE-A cognitive (Frequency Division Duplexing (FDD)) [13].

In [26], authors investigate the coexistence of multiple heterogeneous secondary networks, independently operating in TVWS without a cooperation mechanism. Although interference among heterogeneous networks is also common for other services, e.g., Wi-Fi, Bluetooth, the better propagation conditions in the UHF band, and deployment of regional WRANs increase the complexity of the interference avoidance problem. In [26], authors propose an algorithm for maximizing the desired secondary network throughput by optimally selecting the spectrum channels in the presence of interference from the primary licensed service and other secondary wireless networks. However, interference to other networks is not solved as no cooperation, or priority mechanism is established. According to [27], IEEE 802.22 networks tend to allocate more and better spectrum resources than IEEE 802.11af operating in a common scenario. Our previous research also found slightly better performance for IEEE 802.22 networks [28]. In [27], the authors proposed a centralized cooperative mechanism for allocating spectrum for heterogeneous networks operating on TVWS, considering IEEE 802.11 af and IEEE 802.22 networks.

Geolocation databases might lead to lower interference between the primary and secondary services and also might work as a common spectrum distribution mechanism for heterogeneous networks, but with the drawback of a lower spectrum usage efficiency. This is due to the fact that information in the databases is not updated in real-time [28].

2.3. Algorithms for Channel Selection and Interference Mitigation. In [29], authors present a hierarchically based mechanism for granting fairer spectrum access, based on the kind of wireless service and a reputation-based score. Users that access the spectrum in a selfish way receive a lower score and further lower priority by the centralized allocation system [29]. In [30], a channel selection algorithm for maximizing the users' QoS is proposed. However, it is assumed that the channel needs to be configured for maximum capacity transmission. Our research in [28] demonstrates that the highest MCS is not always the optimal solution as it depends on several factors, including the propagation environment, network load, and the ratio between served traffic and network coverage.

Most researches on coexistence and interference in TVWS consider the signal threshold for reusing a specific channel as a fixed value, for interference from the primary service or another cognitive BS or network. However, a better trade-off between interference and spectrum usage could be achieved if a flexible range of interference allowance is con- sidered, and as such, the interference is dynamically managed. For instance, assigning to a specific user(s), a channel with higher interference might result in a better spectrum usage efficiency and lower mean interference when assessing both Key Performance Indicators (KPIs) for the whole network. For this implementation, centralized access control is required, rather than a distributed spectrum allocation system. This is because the data reported by all users and BSs need to be assessed for finding an optimal configuration for each network device and the whole network performance.

\section{Method}

For minimizing the interference between the primary (broadcasting) and secondary (LTE-A) services and reducing the spectrum usage by the cognitive radio network, we developed a dynamic optimization algorithm for these Key Performance Indicators (KPIs). Further, the software optimization results need to be assessed in a real scenario. In this section, we present the dynamic optimization algorithm for cognitive radio-based networks and the methodology for its experimental validation. We emulate the coexistence scenario and perform measurements for quantifying the impact on the broadcasting service QoE. Figure 1 shows a chart illustrating the optimization and validation process.

First, we define a rural and suburban scenario where a broadcasting primary licensed service (DTMB or DVBT2) coexists with a secondary wireless communication service based on cognitive radio (LTE-A). Here, the service's demands, setup, technological specifications and configurations, and the propagation models are defined (Figure 1(a)). These are the inputs for the LTE-A network dynamic optimization by our multiobjective heuristic algorithm (Figure 1(b)). 'The dynamic optimization of the user's connections to the LTE-A BSs reduces either the spectrum usage, the interference (i.e., LTE-A to broadcasting), or both KPIs. The optimization process generates an interference profile containing the average spatial interference among all transmitters in the whole area. Based on the maximum allowable interference produced by a traditional cognitive radio network for each scenario and user distribution, the optimization gain $(\mathrm{dB})$ in terms of interference mitigation can be calculated. This is the input for an experimental emulation in a real wireless scenario by means of self-developed testbed hardware (see Figure 1(c)). In the context of this research, the emulation is defined as an experimental model where some functional part of it is carried out by a part of the real system [31]. Here, the channel power of a real on-the-air LTE-A signal is reduced in real-time according to the optimization gain at a certain timestamp (obtained from the software dynamic network modelling). For the emulation, we investigate the worst-case scenario at the coverage edge of the broadcasting transmitters in our scenarios (most vulnerable broadcasting signal). The impact of the LTE-A network interference to the broadcasting service QoE is investigated by means of subjective experimental measurements (in Figure 1(d)). In the following subsections, we describe each process (Figures $1(\mathrm{a})-1(\mathrm{~d})$ ) in detail.

In the following subsections, each step of the optimization and experimental evaluation process is described. In 


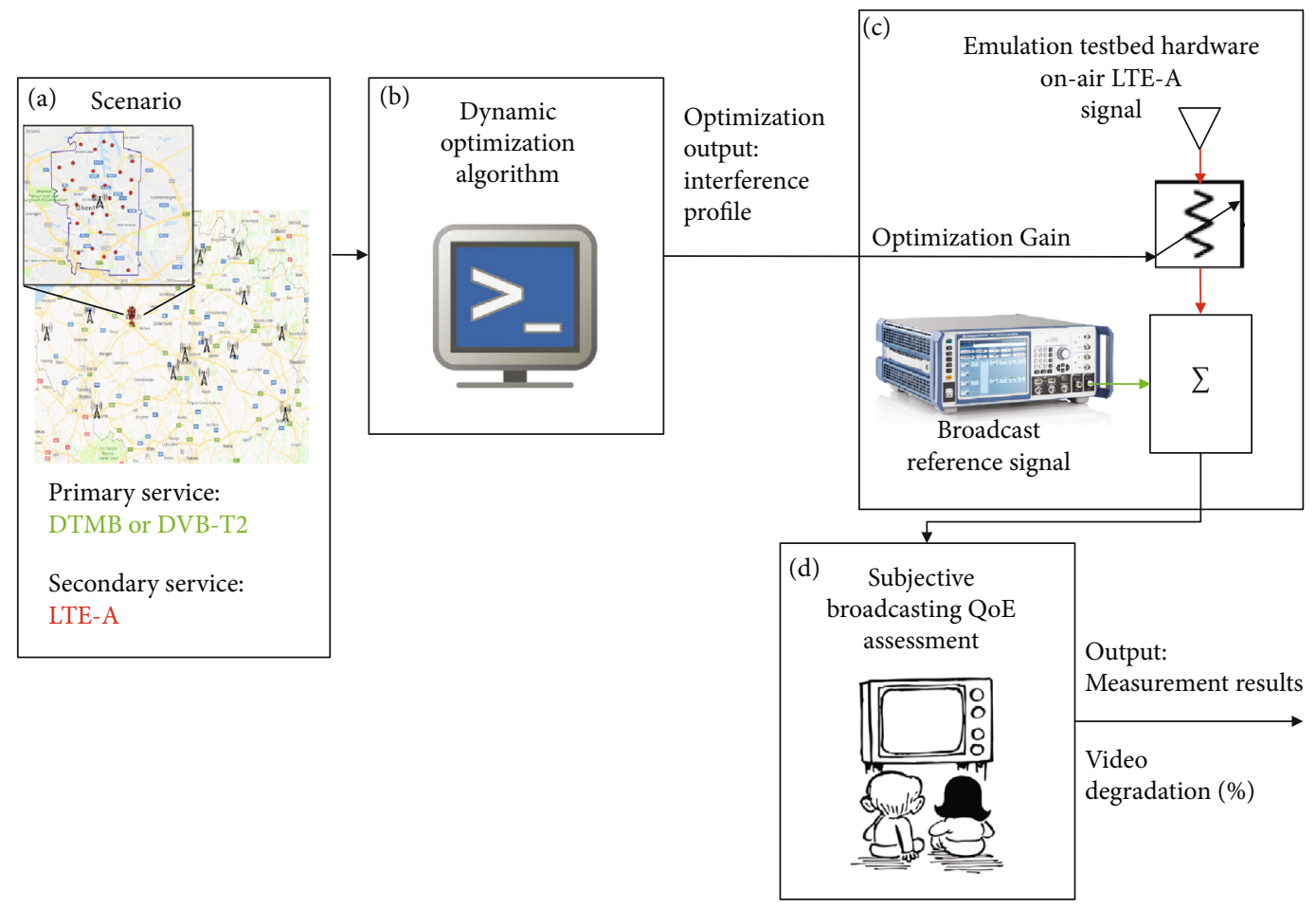

FIGURE 1: Optimization process chart: (a) scenario, (b) optimization algorithm for the LTE-A radio cognitive network, (c) emulation, (d) broadcasting service: subjective QoE measurement.

Subsection 3.1, we detail the characteristics of the scenario, and in Subsection 3.2, the link budget and considered path loss models for propagation calculations (see Figure 1(a)). In Subsection 3.3, the algorithm for the LTE-A dynamic network optimization (see Figure 1(b)) is described. Finally, in Subsection 3.3.1, the broadcasting service experimental interference assessment is explained. This subsection includes the details of the testbed hardware, the emulation conditions, and the QoE assessment (Figures 1(c) and 1(d)).

3.1. Scenario Modelling. We considered two typical scenarios for modelling the interference between the broadcasting and LTE-A network operating on TVWS. Figure 2(a) shows a rural scenario in the outskirts of Havana, Cuba, where a Multifrequency Network (MFN) operates using the DTMB broadcasting transmission standard [32]. Figure 2(b) shows a suburban scenario in Ghent, Belgium, where different Single-Frequency Networks (SFN) operate according to the DVB-T2 broadcasting transmission standard [33].

In the rural area where the LTE-A network provides services (delimited by the blue line in Figure 2(a)), the average population density is approximately 200 people per square kilometer. 10 LTE-A BSs (marked with red dots in Figure 2(a)) serve the traffic generated at the peak time being, on average $135 \mathrm{Mbps}$. In the suburban scenario in Ghent (delimited by the blue line in Figure 2(b)), the population density is 1700 people per square kilometer, and the peak traffic generated is, on average, $224 \mathrm{Mbps}$, being served by a total of 27 BSs.

All the broadcasting transmitters in and surrounding the areas where the LTE-A cognitive networks operate (black icons in Figures 2(a) and 2(b)) are included for assessing the interference and dynamically managing the spectrum to be used by the LTE-A network. There are 16 broadcasting towers using 16 different frequencies in the suburban scenario and 15 broadcasting towers using 11 different frequencies in the rural area. In the rural scenario, there is at most a single standard definition stream and a single high definition stream per transmission tower. In the suburban scenario, there are up to 5 services per tower, leading to a higher density of spectrum usage.

3.2. Link Budget and Path Loss Models. Table 1 lists the specifications of the DTMB, the DVB-T2 broadcasting transmitters, and the LTE-A BSs.

The LTE-A EIRP corresponds with the maximum radiating power allowed by most regulatory domains for the operation of secondary cognitive radio devices in TV white spaces $[13,22]$. For Ghent LTE-A network planning, we consider an $8 \mathrm{MHz}$ channel bandwidth based on the carrier aggregation mode. We include the aggregation of a $3 \mathrm{MHz}$ and a $5 \mathrm{MHz}$ channel. Hence, the OFDM specifications correspond with the equivalent for the aggregated carriers in this channel configuration. For the Havana LTE-A network, a channel of $5 \mathrm{MHz}$ is considered. The OFDM carriers specifications for LTE-A are the ones defined in [34], for DTMB in [32], and for DVB-T2 in [33]. The frequencies of each LTE-A BS are dynamically assigned within the specified range by the network optimization algorithm.

The DTMB and DVB-T2 specifications correspond with the technological settings for the actual broadcasting transmissions in the areas under test. The current frequency of 
(b) Suburban scenario, Ghent

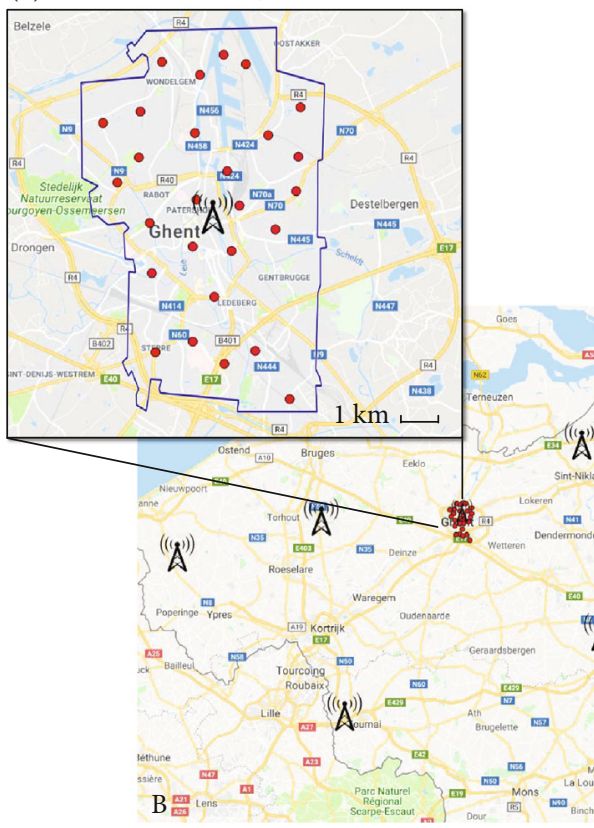

(a) Rural scenario, Havana outskirts

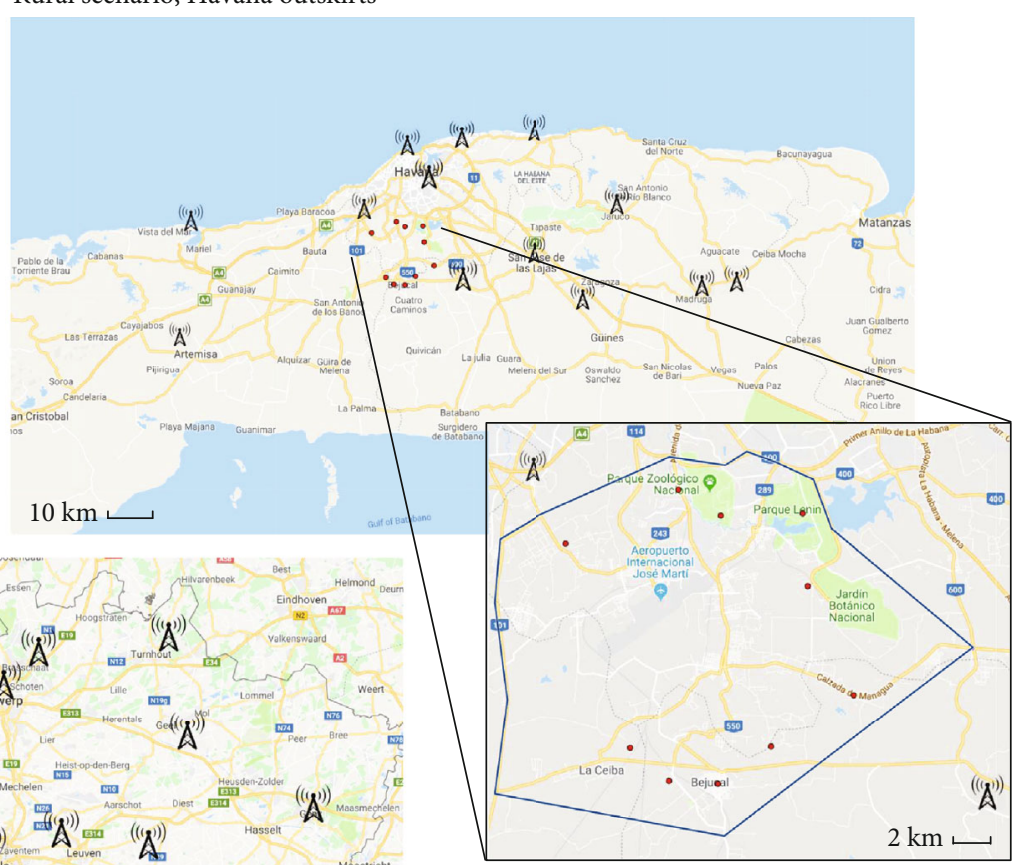

FIgURe 2: Network scenarios: (a) LTE-A coexisting with DTMB broadcasting networks in a rural scenario in Havana, (b) LTE-A coexisting with DVB-T2 broadcasting networks in a suburban scenario in Ghent. LTE-A BSs are marked with red dots; TV broadcasting towers are marked with black icons; the area for the LTE-A network service is delimited by a solid blue line.

each broadcasting transmitter in the region is considered for the propagation and interference calculations. Noise figure and sensitivity correspond with the testbed hardware specifications for such settings (details on the testbed hardware are provided in Section 3.4.4). Notice that shadow margin and fade margin for DTMB and DVB-T2 are higher because we include the broadcasting transmitters in the surrounding of the areas under test with different propagation conditions. The protection margins are assumed according to the recommendations in TS 136104 Release 12: LTE; Evolved Universal Terrestrial Radio Access (E-UTRA); base station (BS) radio transmission and reception [35].

For assessing the interference to and from the broadcasting transmitters, all the transmitters in the area and surrounding the LTE-A cognitive network are considered up to a distance of approximately $120 \mathrm{~km}$. First, the path loss for accounting for the propagation losses experienced by the signal in the environment has to be quantified. Equation (1) defines a generic function for accounting for the path loss in any environment [36].

$$
\mathrm{PL}=g\left(d, f, h_{\mathrm{tx}}, h_{\mathrm{rx}}\right)
$$

where PL $(\mathrm{dB})$ is the path loss, $f(\mathrm{MHz})$ is the transmission frequency, $d(\mathrm{~km})$ is the distance between the transmitter $(\mathrm{tx})$ and the receiver ( $\mathrm{rx})$, and the transmitter and receiver heights are denoted by $h$. The function $g($.$) will depend on$ the propagation model that fits the propagation characteristics of the investigated scenario [36]. For defining $g($.$) , differ-$ ent propagation models have been proposed in the literature and further standardized. These models account for environmental factors, e.g., the terrain topology, the ground characteristics, and obstacles. For the rural scenario in the Havana outskirts, we consider the Okumura-Hata path loss model for rural environments [37], as this model showed a better fit than the ITU-R P.1546 [38] model according to a largescale measurement campaign in the rural area of Havana [39]. The ITU-R model predicts much lower propagation losses over near-flat terrain without significant obstacles compared to the actual measurements. Notice that most of the land use in our rural scenario consists of farm lands and small community houses. For the suburban scenario in Ghent, an empirical one-slope path loss model was determined based on a large-scale measurement campaign in Ghent [40]. This model was designed based on a large-scale measurement campaign in Ghent city for a reference frequency of $602 \mathrm{MHz}$. For reducing the error when accounting for other frequencies $f(\mathrm{MHz})$, we apply a correction factor $\varepsilon_{f}$ as defined in

$$
\varepsilon_{f}=20 \log \left(\frac{f}{602}\right) .
$$


TABLE 1: Link budget parameters.

\begin{tabular}{|c|c|c|c|c|}
\hline Parameter & Cognitive LTE-A & DTMB & DVB-T2 & Unit \\
\hline Transmitter EIRP & 36 & $60.5-77.5$ & $71-82.5$ & $\mathrm{dBm}$ \\
\hline Maximum allowable interference signal level (from specified technology) & -97 & -95 & -95 & $\mathrm{dBm}$ \\
\hline Frequency & $\begin{array}{l}470-698 \\
470-790\end{array}$ & $470-698$ & $470-790$ & $\mathrm{MHz}$ \\
\hline Bandwidth & $\begin{array}{c}8(3+5) \\
\mid 5\end{array}$ & 6 & 8 & $\mathrm{MHz}$ \\
\hline Total subcarriers & $\begin{array}{c}482(181+301) \\
\mid 301\end{array}$ & 3780 & 32768 & - \\
\hline Used subcarriers & $\begin{array}{c}768(256+512) \\
\mid 512\end{array}$ & 3744 & 27841 & - \\
\hline Frequency sampling factor & 1.536 & 0.945 & 0.9712 & - \\
\hline Modulation scheme & Variable & 64-QAM & 64-QAM & - \\
\hline Coding scheme & Variable & 0.6 & $3 / 4$ & - \\
\hline BS antenna height & $20-50$ & $80-262$ & $64-302$ & $\mathrm{~m}$ \\
\hline Cell interference margin & 2 & 0 & 0 & $\mathrm{~dB}$ \\
\hline Receiver antenna height & $1.5-3$ & 3 & 3 & $\mathrm{~m}$ \\
\hline Receiver antenna gain & 11.5 & 8 & 11.5 & $\mathrm{~dB}$ \\
\hline Receiver feeder losses & 0.5 & 0.5 & 0.5 & $\mathrm{~dB}$ \\
\hline Noise figure & 7 & 7 & 6 & $\mathrm{~dB}$ \\
\hline Receiver minimum sensitivity & -90.5 & -84.5 & -83.5 & $\mathrm{dBm}$ \\
\hline Shadow margin & $8 \mid 7$ & 12.3 & 12.3 & $\mathrm{~dB}$ \\
\hline Fade margin & 7.4 & 8 & 9 & $\mathrm{~dB}$ \\
\hline
\end{tabular}

The Ghent model cannot be used in its surroundings from $10 \mathrm{~km}$ to $120 \mathrm{~km}$. This is because the Ghent model is valid up to $10 \mathrm{~km}$. Hence, in our application, within Ghent, the oneslope model is used, and for the broadcasting transmitters outside the Ghent area, the ITU-R P.1546 model is used.

Once the path loss is known, the signal level at any given location for all radiating devices can be quantified, taking into account the specified technological specifications of the transmitters and additional environmental factors. This allows for accounting for the interference margin among services using the same frequency, i.e., carrier signal to interference signal ratio $\mathrm{C} / \mathrm{I}(\mathrm{dB})$. The coverage of a particular service (coverage radius) $R(\mathrm{~km})$ is defined by the maximum allowable path loss $\mathrm{PL}_{\max }(\mathrm{dB})$, the environmental propagation function, and a certain margin for accounting for the signal fading $F_{d}(\mathrm{~dB})$ and shadowing $S_{w}$ [36]. Equation (3) defines the coverage radius as a function of the maximum allowable path loss.

$$
R=g^{-1}\left[\left(\mathrm{PL}_{\max }-F_{d}-S_{w} \mid f, h_{\mathrm{tx}}, h_{\mathrm{rx}}\right) .\right.
$$

Notice that the maximum allowable path loss depends on the technology specifications, including the transmitters' and receivers' setup. The link budget defining the technologies' specifications and configuration for accounting for $\mathrm{PL}_{\max }$ is listed in Table 1.

3.3. Cognitive LTE-A Network Optimization in TV White Spaces. For the cognitive wireless network dynamic optimization, we base ourselves on the heuristic capacity-based opti- mization algorithm GRAND (Green Radio and Access Network Design) [41] with the modifications for cognitive radio-based technologies described in [28]. The algorithm takes a set of users and traffic densities as input parameters and manages the user connections to the BSs, the radiated power, and the BSs' operational modes (idle/active) for optimizing a certain network parameter, e.g., network power consumption [41] [28]. However, the algorithm does not optimize the spectrum usage taking into account a dynamic interference assessment on a case-by-case basis. The KPIs considered here are network interference and spectrum usage.

In laboratory experiments for LTE-A BSs of three different service providers, we have observed that the LTE-A BSs can maintain the users' mean throughput degradation below $25 \%$ by dynamically managing the MCS and the PRBs (Physical Resource Blocks) among different users connected to the same cell. For both DTMB and DVB-T2 interference to the LTE-A network, the interference can be coped within a range of 3 to $5 \mathrm{~dB}$. A throughput degradation of $25 \%$ corresponds with a Data Block Error Rate (DBER) lower than 10\%, for which the experimental interference assessment in LTE networks is recommended in ITU-R BT.2215-7 Report [42]. The interference management depends mainly on the mobile network operator connection management and the signaling time (update time). By dynamically managing the interference in our optimization algorithm in a range of $5 \mathrm{~dB}$ from the maximum allowable interference, it is possible to achieve a better network spectrum usage and lower average network interference without significantly affecting the QoS of the wireless network. 


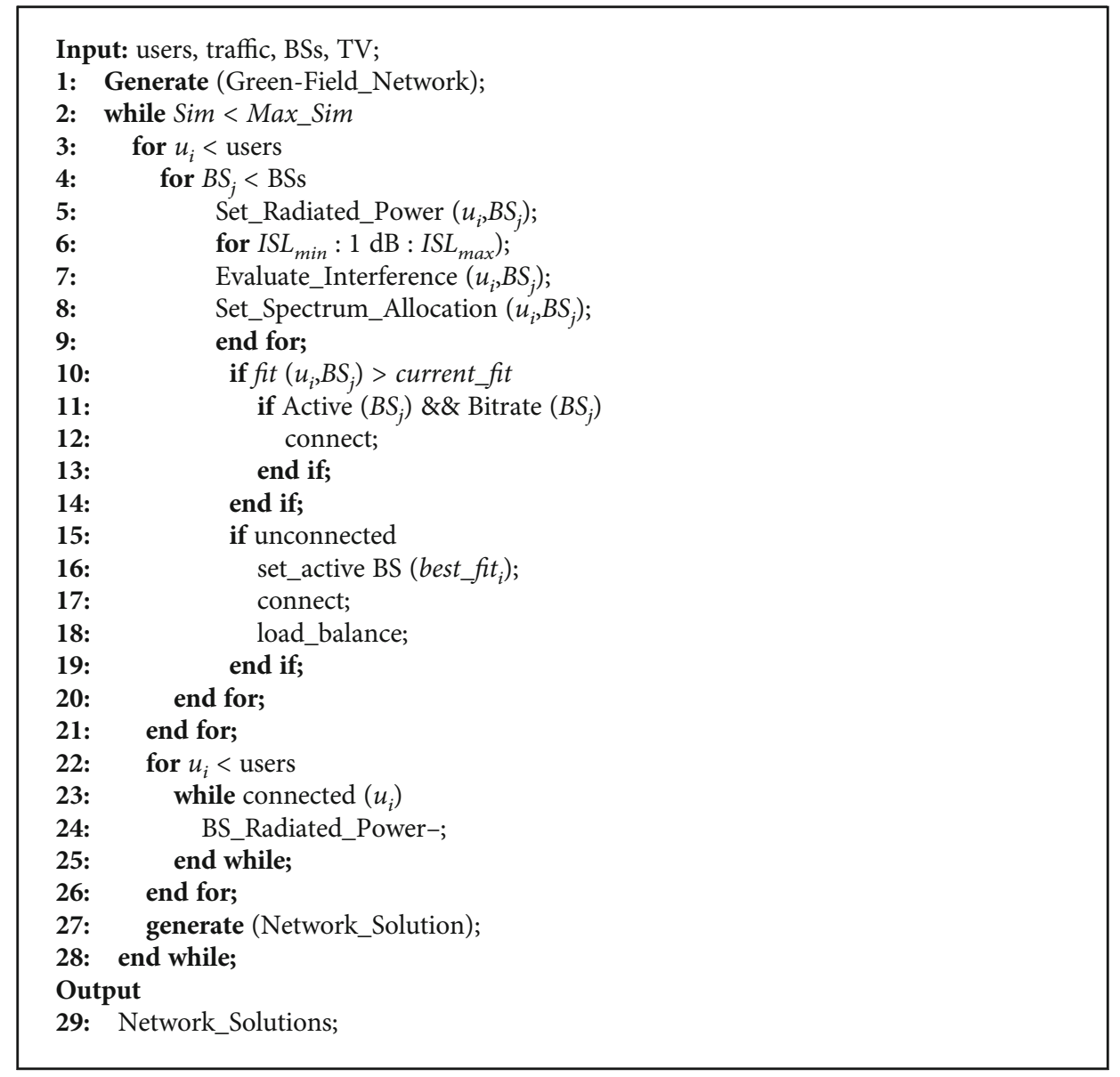

Algorithm 1: Optimization algorithm for minimizing interference and reducing spectrum usage.

3.3.1. Dynamic Network Optimization Algorithm. Algorithm 1 describes the dynamic optimization algorithm for the cognitive radio LTE-A network. This algorithm allows minimizing the interference between services and achieving a higher spectrum usage efficiency, without affecting the wireless network QoS. By reducing the interference to the broadcasting network, the TV service users' QoE is improved.

The algorithm is heuristic and capacity based. The inputs are the user density, the traffic demand, the cognitive LTE-A, and the TV Broadcasting station specifications (see Table 1) and its geolocations (see Figure 2).

First, based on the input parameters and constraints, the algorithm generates a green-field network planning with the initial settings and locations of the BSs and TV stations. The users are randomly and uniformly distributed over the whole area (line 1 in Algorithm 1). This means each pixel in the map has the same probability of having a user requesting a connection to the network.

The algorithm runs for a total number of simulations (Max_Sim in line 2, Algorithm 1). The number of simulations is defined by the progressive average of the network KPIs, i.e., average network interference and spectrum usage. Here, we consider that if the progressive average of any KPI has a standard deviation higher than $5 \%$, the number of sim- ulations is not high enough. This is correlated with the proportion of the number of users and the area size and the random nature of the user allocations. The lower the number of users per square kilometer, the more diverse the possible distributions of those users, and the higher the number of possible solutions. As the algorithm is heuristic, the best solution is not found but a solution good enough for satisfying the defined constraints. Hence, for a higher degree of freedom and possible solutions, a higher number of simulations are required.

For each user $u_{i}$ in the area, the algorithm tries to find the connection to the cognitive LTE-A base station $\mathrm{BS}_{j}$ that best suits the network KPI optimization goals. First, for $\mathrm{BS}_{j}$ 's maximum coverage range, the algorithm seeks if the $u_{i}$ is located in its range. In such a case, the algorithm will analyze for each channel the interference from all other LTE-A BSs and TV broadcasting stations in the area. The interference signal level (ISL) threshold will be dynamically moved in a range from $\mathrm{ISL}_{\min }$ to $\mathrm{ISL}_{\max }$ with a resolution of $1 \mathrm{~dB}$. The $\mathrm{ISL}_{\max }$ is defined in Table 1 , Section 3.2 , and $\mathrm{ISL}_{\min }$ is $5 \mathrm{~dB}$ lower. The spectrum allocation function will reduce the spectrum usage for each ISL constraint (lines 6 to 9 in Algorithm 1). The interference from all broadcasting stations and LTE-A devices at $u_{i}$ and $\mathrm{BS}_{j}$ geolocation is calculated by the path loss models defined in Section 3.2. If the interference is 
lower than the current ISL constraint, the connection at the channel being analyzed can be stablished. Otherwise, a new channel is analyzed. Lower frequencies in the spectrum are tried first because of their lower path loss attenuation, the radiated power can be decreased, and as a consequence, the interference and the network power consumption are reduced.

3.3.2. KPI Optimization within the Algorithm. The final connection of $u_{i}$ to $\mathrm{BS}_{j}$ among all $j$ possibilities is determined by a fitness function that balances both network KPIs: interference and spectrum usage (line 10 in Algorithm 1). The network fitness function is defined by

$$
\begin{aligned}
\operatorname{fit}\left(u_{i} ; \mathrm{BS}_{j}\right)= & w_{1} \cdot\left(1-\frac{S_{U}}{S_{\max }}\right)+w_{2} \\
& \cdot\left[1-\frac{1}{2}\left(\frac{\mathrm{ISL}_{\mathrm{TV}}}{\mathrm{ISL}_{\mathrm{TV} \max }}+\frac{\mathrm{ISL}_{\mathrm{A}}}{\mathrm{ISL}_{\mathrm{Amax}}}\right)\right],
\end{aligned}
$$

where ISL $\mathrm{A}_{\mathrm{A}}$ is the interference caused by the LTE-A BSs and ISL $_{\mathrm{TV}}$ is the interference caused by the TV broadcasting stations. These values are normalized to the maximum interference for the TV broadcasting transmitters $\left(\mathrm{ISL}_{\mathrm{TVmax}}\right.$ ) and the LTE-A BSs (ISL Amax $_{\text {m }}$. The spectrum usage $S_{U}$ is the number of channels being required in the area of the $u_{i}$; $\mathrm{BS}_{j}$ connection is settled and is normalized by the maximum number of channels in the band $S_{\max }$. The allowed interference has an inverse relationship with the spectrum usage. For a lower interference threshold, a higher number of channels will be used by the network. Hence, the weights $w_{1}$ and $w_{2}$ are used for balancing the relevance of both KPIs for the final network optimization solution. Here, we analyze three cases of weight combinations, i.e., $\left(w_{1} ; w_{2}\right)=\{(1 ; 0),(0.5 ; 0.5),(0 ; 1)\}$.

Each normalized parameter in the fitness function responds to the average network performance. Hence, the user $u_{i}$ will be connected to the $\mathrm{BS}_{j}$ and in the channel that best fits the network KPI optimization goals rather than maximizing the user KPI or QoS itself. Figure 3 shows an example of a user connection $\left(u_{x}\right)$ to the best solution from the entire network's point-of-view, instead of the user's point-of-view. In this example, the connection of $u_{x}$ to $\mathrm{BS}_{1}$ (operating frequency $f_{1}$ ) maximizes its effective throughput by using 64QAM. However, in terms of interference to the broadcasting service, this is not the best network solution. The algorithm might choose the $\mathrm{BS}_{2}$ (operating frequency $f_{2}$ ) for connecting $u_{x}$, depending on the interference weight in the fitness function. Hence, it could happen that a user is connected to a channel that is not the best for this particular user, but by doing so, the overall network interference is reduced, or other channels can be reused by other devices, leading to higher overall network performance. The established connection must satisfy the user traffic demand and a minimum QoS threshold.

For satisfying the QoS requirement, the allowed interference is limited. The interference is low enough for allowing the users to satisfy their traffic demand with some of the available MCS (e.g., QPSK in the example of Figure 3). In addition, a minimum number of LTE-A BSs are activated depending on the network load and coverage requirements

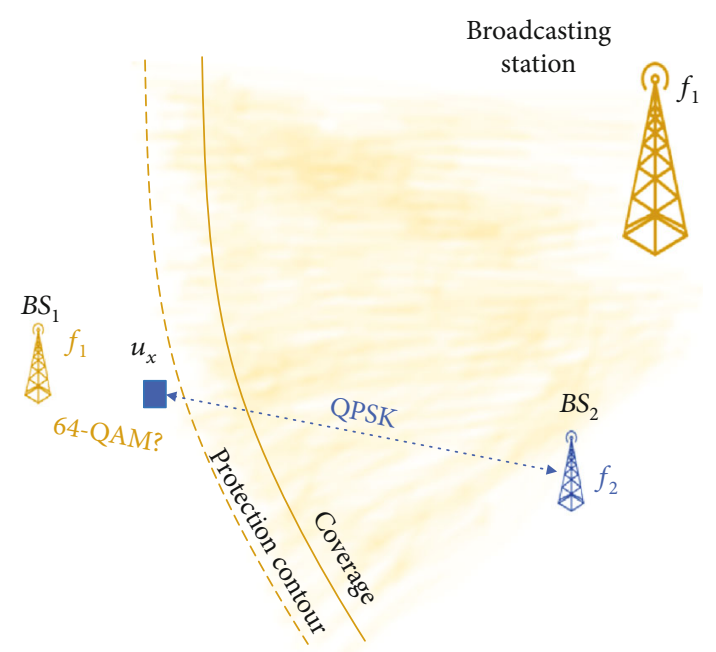

Figure 3: Connection of a hypothetical user $u_{x}$ to the best BS for optimizing the network KPIs.

(lines 11 and 12 in Algorithm 1) [28]. In this way, other relevant network parameters like power consumption can be minimized. Nevertheless, if one of the active BSs cannot serve the user traffic demand, because of capacity, coverage, or interference constraints, a new BS is activated. The algorithm will seek the BS with the highest fitness and will try to balance the network load by moving users to different BSs if this allows the current user connection, and the network fitness is not decreased (lines 15 to 19 in Algorithm 1).

A further reduction in the radiated power will lead to a lower interference, as long as the connection is maintained (line 22 to 26 in Algorithm 1), i.e., the experienced path loss to the $\mathrm{BS}$ is lower than the maximum allowable path loss $\left(\mathrm{PL}<\mathrm{PL}_{\max }\right.$, see Section 3.2). Also, the reduction of the radiated power and number of active BSs causes a reduction in network power consumption.

Finally, an optimal network solution for each simulation is generated. For each simulation, the software retrieves the spectrum usage distribution and an interference profile, including the achieved optimization gain $G_{O}(\mathrm{~dB})$ for this KPI. The optimization gain is calculated by means of the following equation:

$$
G_{O}=\mathrm{ISL}_{\max }-\mathrm{ISL}_{50} .
$$

Here, $\mathrm{ISL}_{50}$ is the mean interference signal level in the whole area for a certain frequency. For the analysis of the results and for performing the experimental emulation (details in the following subsections), we consider the channels with the highest interference (worst-case).

3.4. Broadcasting Service Experimental Interference Assessment. Figure 4 describes the interference conditions that we want to emulate in a real area with certain controlled variables, i.e., guarantee the desired broadcasting signal level at least $99 \%$ of the time. Here, our goal is to investigate the critical conditions for which a certain frequency $f_{1}$ can be reused, the caused interference and its impact on the 
broadcasting users QoE. We also investigated the impact of the $G_{O}$ for reducing the harmful interference to the broadcasting network receivers.

This model is based on the average emission footprints of the local broadcasting stations, i.e., average height, radiated power, and propagation conditions. Here, our goal is to emulate the critical conditions (worst-case) for the broadcasting network being interfered with by the cognitive LTE-A network. Hence, the subjective test is performed for the same conditions at the coverage edge of the broadcasting station (see the solid line and marker subjective QoE test in Figure 4). The protection area of the broadcasting transmitter will increase depending on the achieved optimization gain for the interference KPI (see Equation (5) in Section 3.3.2). The worst-case corresponding with $0 \mathrm{~dB}$ optimization (at ISL $=-95 \mathrm{dBm}$ ) is denoted with a red dashed line in Figure 4). The higher the optimization gain for the interference KPI, the higher the protection area radio and the higher the path loss from the LTE-A device to the edge of the broadcasting transmitter (see dotted lines in Figure 4). Hence, a lower interference from the LTE-A devices to the broadcasting network is achieved. In Sections 3.4.2 and 3.4.3, we provide details about the specifications for the suburban and rural scenario models, respectively.

The goal of the subjective QoE experiments is to quantify the perceived quality of the broadcasting service interfered with by an LTE-A cochannel signal. We evaluate the perceived quality of the broadcasting service interfered with by the actual LTE-A signal. We compare the broadcasting video degradation caused by the actual LTE-A on-the-air-signal with a channel power equivalent to the network operating with a fixed ISL threshold versus optimized with the dynamic interference optimization (interference optimization gain, see the algorithm output in Section 3.3).

3.4.1. QoE Subjective Indicators. Two leading subjective indicators have been proposed in the literature for quantifying the threshold for which the objective technical parameters are degraded enough to affect the Quality of Experience of broadcasting service users. Report ITU-R BT.2035-2/2008 defines the Threshold of Visibility (TOV) as part of the guidelines and techniques for the evaluation of digital terrestrial television broadcasting systems in laboratory environments [43]. Hence, the TOV is the threshold where a trained observer notices any kind of pixelation in the image in a time-lapse of 1 minute [43]. Recommendation ITU-R BT.1368-13/2017 defines the planning criteria, including protection ratios, for digital terrestrial television services in the VHF/UHF bands [44]. This recommendation defines the Subjective Failure Point (SFP) as a method for quantifying and defines protection ratio measurements in a unified way. Then, SFP is defined as the quality level, where no more than one error is visible in the picture for an average observation time of 20 seconds [44].

By accounting on how many timestamps the TOV and SFP are exceeded at the broadcasting coverage edge (solid line in Figure 4), we will compare the performance of the developed algorithm for spectrum optimization only, interference optimization only, and an equally balanced strategy for both KPIs considering a flexible range of interference allowance. In addition, we will compare these results with the case of spectrum management by a traditional cognitive radio $\mathrm{BS}$, considering the highest allowable interference (-95 dBm).

Notice that both TOV and SFP have been defined as a criterion to find a limit for a just error-free picture at a TV screen for protection ratio measurements. In the context of our experiment, our goal is not to find the protection ratio limits, but how many times these limits are exceeded. If exceeding these limits causes such BER that more than one pixelation is noticed within the same timestamp, a single assertion is recorded.

3.4.2. Rural Scenario Specifications. In the DTMB rural measurements, the mean broadcasting tower height above average terrain (height in Figure 4) is approximately $111 \mathrm{~m}$. The average EIRP of the broadcasting transmitters (in Figure 4) is $65.4 \mathrm{dBm}$. These parameters are based on the current settings of the operating transmitters and were retrieved from the local service providers. The receiver's theoretical sensitivity for $6 \mathrm{MHz}$ channel bandwidth is $-84.5 \mathrm{dBm}$ (this defines the solid line in Figure 4). In a large-scale laboratory measurement, the average sensitivity throughout the UHF band was $-84 \mathrm{dBm}$, with a standard deviation of approximately $2 \mathrm{~dB}$ [23]. The edge of the broadcasting station is located around $52 \mathrm{~km}$ from the transmitter (solid line in Figure 4). This is the radius for which under the considered propagation conditions, it is guaranteed a signal level higher than $-84 \mathrm{dBm}$ in $90 \%$ of the locations $99 \%$ of the time (Figure 4 , solid line). For the worst-case interference conditions, the protection contour of the broadcasting station is located $60 \mathrm{~km}$ from the coverage edge (dashed line in Figure 4). This value is obtained considering the highest allowable interference, i.e., the mean broadcasting signal level is $-95 \mathrm{dBm}$ (dashed line in Figure 4). The worst interference case corresponds with an LTE BS or CPE radiating the maximum allowable EIRP in the edge of the broadcasting station protection contour for $G_{O}=0 \mathrm{~dB}$ (located in the dashed line in Figure 4). Hence, accounting for the proper path loss, for the worst-case, there is an LTE-A mean interference signal level of $-103.7 \mathrm{dBm}$ at the coverage edge of the broadcasting station with a fading variation of approximately $8 \mathrm{~dB}$ (see marker LTE-A mean interference level over the solid line in Figure 4).

3.4.3. Suburban Scenario Specifications. For Ghent, we also considered the average footprint of the regional DVB-T2 broadcasting transmitters in the area. The average height of the transmitters considered is $112 \mathrm{~m}$ and average EIRP = $74 \mathrm{dBm}$. These parameters are also based on the current settings of the operating transmitters in the region. Here, we considered only the transmitters with an EIRP $<75 \mathrm{dBm}$ and height above the average terrain below $200 \mathrm{~m}$. For the transmitters with higher EIRP and/or higher height above the average terrain, the mean ISL from the LTE-A devices is lower than $-129 \mathrm{dBm}$ (LTE-A interference over the solid line in Figure 4). As a consequence, a critical harmful interference condition is never reached. For the DVB-T2 


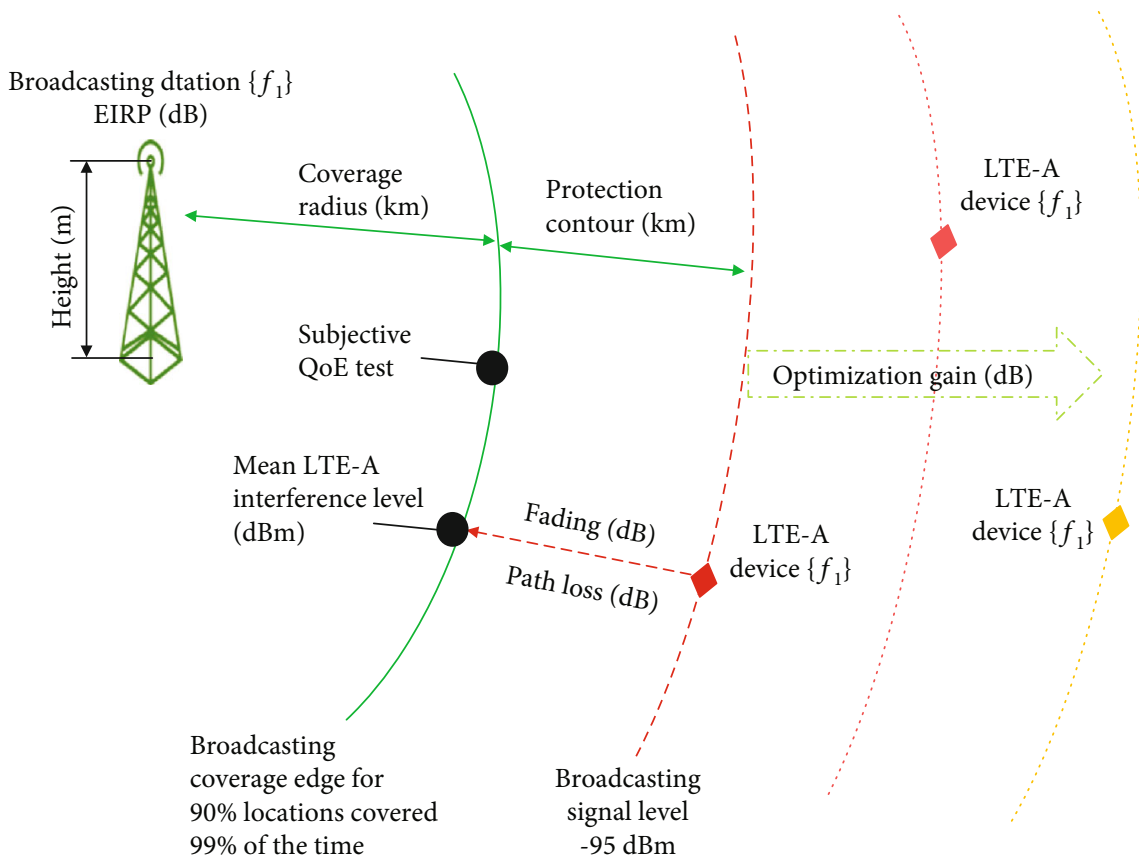

Figure 4: Emulation scenario model. The solid line defines the coverage edge of the broadcasting transmitter for $90 \%$ of locations covered, 99\% of the time, the dashed line defines the limit of the broadcasting transmitter protection contour for the worst-case interference, and the dotted lines represent the extension of the broadcasting transmitter protection contour as a result of the algorithm optimization gain for the interference KPI. The diamond markers represent possible LTE-A devices reusing the same frequency $f_{1}$.

modulation and coding scheme defined in the link budget, the minimum broadcasting signal level is $-82.5 \mathrm{dBm}$. Here, we consider an additional margin of $0.5 \mathrm{~dB}$, being the broadcasting signal level for the experiments $-83 \mathrm{dBm}$ (minimum broadcasting signal level, the solid line in Figure 4). The coverage radius of the broadcasting service is then just $17.9 \mathrm{~km}$, and the protected contour is located another $12.5 \mathrm{~km}$ away from the coverage edge (for the interference worst-case, being the optimization gain equal to $0 \mathrm{~dB}$ ). It is important to mention that the difference in coverage and the protected areas between the rural and the suburban scenario is not because of the technologies link budget differences but because of the propagation environment (different propagation models). Then, for the DVB-T2 worst-case $\left(G_{O}=0 \mathrm{~dB}\right)$, the mean interference from the LTE-A cognitive network in the coverage edge of the broadcasting transmitter is $-105.7 \mathrm{dBm}$ for the critical conditions described for this scenario (LTE-A interference marker over the solid line in Figure 4).

3.4.4. Testbed Hardware. We designed testbed hardware for emulating as close as possible to the reality the scenario described in Figure 4. Figure 5 shows a diagram of the designed testbed hardware for the experiments.

An on-the-air LTE-A signal is filtered and translated in frequency to the UHF band. A UHF band filter attenuates $(>30 \mathrm{~dB})$ the signals generated out of the band for avoiding causing interference to local radio services. The return loss is higher than $18 \mathrm{~dB}$. A step attenuator is configured at a fixed attenuation of $19 \mathrm{~dB}$ or $34 \mathrm{~dB}$ for having an interference signal at the receiver with a mean level (50-percentile) of $-104 \mathrm{dBm}$ (mean LTE-A interference in Figure 4 and also see spectrum analyzer in Figure 5) for the rural scenario and $-106 \mathrm{dBm}$ for the suburban scenario. The signal channel power is measured and averaged over a period of 1 minute with a sampling rate higher than $26 \mathrm{~Hz}$, as recommended in [45].

A field-programmable gate array (FPGA) (see Figure 5) is loaded with the attenuation values relative to the difference between the optimized interference solutions generated by GRAND at each time stamp and the maximum allowable interference in a traditional cognitive radio network (optimization gain). The FPGA transcodes this value to the equivalent attenuation settings in the testbed hardware. The maximum allowable interference at the edge of the protection contour of the TV broadcasting signal is $-95 \mathrm{dBm}$ (see dashed line in Figure 4). For instance, if the optimization algorithm reduces the interference on average $3.1 \mathrm{~dB}$ for a certain simulation, the FPGA will configure the attenuator for having a reduction of the channel power by $3 \mathrm{~dB}$. The attenuator in our hardware has a resolution of $0.5 \mathrm{~dB}$. Hence, any value will be approximated to the closest fraction of $0.5 \mathrm{~dB}$. Then, in the rural scenario, the LTE-A mean interference signal level will be reduced from $-104 \mathrm{dBm}$ to $-107 \mathrm{dBm}$. However, these are mean values. In the experimental testbed, the signal will be affected by the real fading during the measurements. Therefore, the equivalent interference signal level will be $-107 \mathrm{dBm} \pm 8 \mathrm{~dB}$ for this specific optimization output.

The LTE-A interference signal is added by a directional combiner to a controlled digital television signal (DTMB or DVB-T2), generated by the R\&S Broadcast Test Center (in Figure 5). Here, the broadcasting video signal is locally 

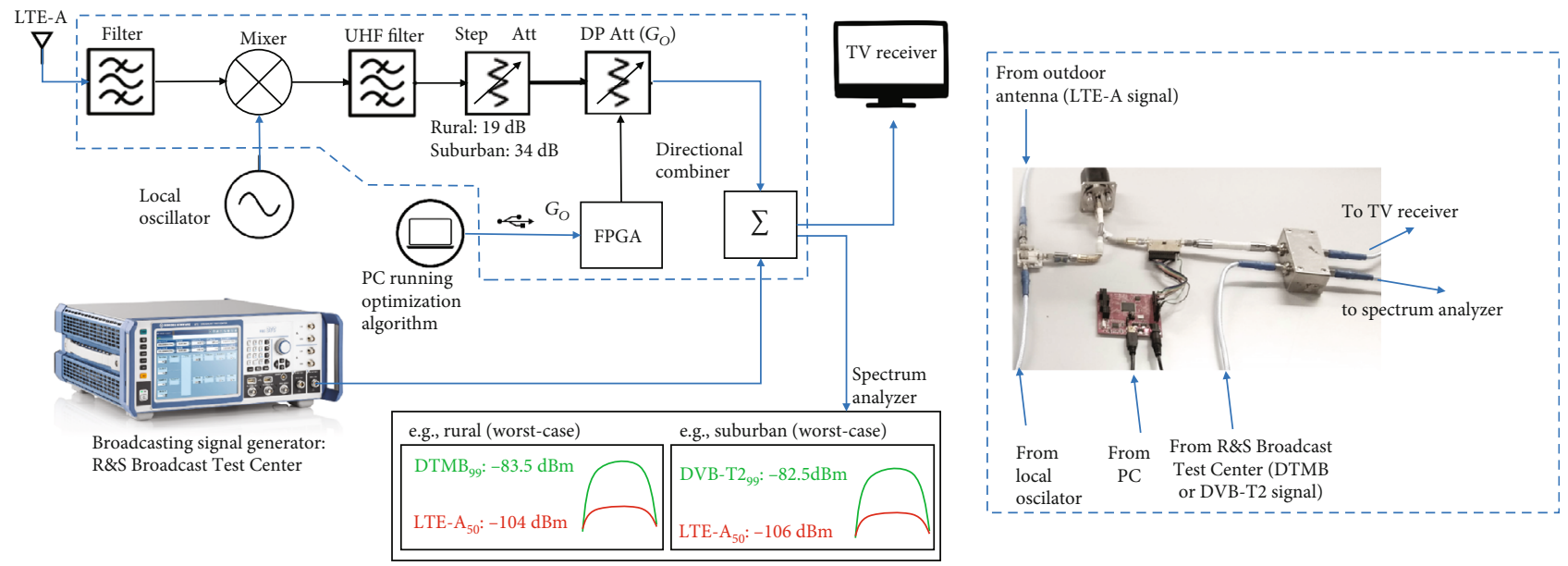

FIGURE 5: Testbed hardware diagram including a tuning antenna receiving the on-the-air LTE-A signal, a band pass filter, a downconverter mixer, and its local oscillator, a UHF band filter, a step attenuator (Step Att), and a Digital Programmable Attenuator (DP Att), a Field Programmable Gate Array (FPGA) connected through USB to a computer (PC) running our algorithm, a Directional Combiner, a Broadcasting signal generator, a TV Receiver, and a spectrum analyzer.

generated to guarantee the desired signal level more than 99\% of the time. This matches the defined coverage contour of the broadcasting transmitter (solid line in Figure 4). The R\&S Broadcast Test Center configuration is listed in Table 2. For both scenarios, the broadcasting signal channel power is $+0.5 \mathrm{~dB}$ above the threshold defined for the coverage edge of the transmitter (solid line in Figure 4).

The LTE-A signal mean channel power is monitored in time windows of 10 minutes and averaged throughout $1 \mathrm{~min}$ ute to avoid and detect any significant change in the propagation conditions or more substantial fading variations. This is performed to guarantee that the initial system conditions are maintained throughout the test.

For assessing the variations on QoE by means of the TOV, a trained observer will assert 1 if at least one pixelation or distortion is noticed in any timestamp of 1 minute. The asserted timestamps indicate the percentage of timestamps the TOV is exceeded. In a similar way for the SFP metric, a trained observer will assert 1 if at least one pixelation or distortion is noticed in any timestamp of 20 seconds. The asserted timestamps indicate the percentage of timestamps the SFP is exceeded. The observer is located at a recommended distance of $1.2 \mathrm{~m}$ to $1.8 \mathrm{~m}$ from the TV receiver (in Figure 5). The total observation time is 40 minutes divided into 4 sets of 10 minutes for avoiding visual fatigue, minimizing the probability of missing the occurrence of a noticeable picture distortion.

\section{Results}

4.1. Dynamic Network Optimization Results. Figure 6 shows the optimization results for the required spectrum for the broadcasting and LTE-A networks in each scenario and the equivalent average interference signal levels. These results correspond to the KPI weights defined in Section 3.3.

The highest ISL from the broadcasting network is reached when the algorithm optimizes SU without taking into account the interference metric for deciding LTE-A user connections (markers $2 \mathrm{a}$ and $4 \mathrm{a}$, in Figure 6). In the rural scenario, the highest average ISL from the broadcasting DTMB signal is $-98.5 \mathrm{dBm}$ (marker 2a in Figure 6). For the suburban scenario, the ISL of the broadcasting DVB-T2 signal is approximately $1.1 \mathrm{~dB}$ higher (marker $4 \mathrm{a}$ in Figure 6). This difference is due to the fact that in the rural scenario with a lower traffic per square kilometer, more spectrum can be reused despite the fact that a better propagation might increase the interference from the surrounding broadcasting towers. Indeed, for this optimization case, the spectrum usage in the rural scenario is, on average, approximately 1.7 channels lower than in the suburban scenario (markers 1a and $2 \mathrm{a}$ compared with markers $3 \mathrm{a}$ and $4 \mathrm{a}$ in Figure 6).

When the interference KPI weight is maximum $\left(w_{2}=1\right)$, the algorithm leads to an average ISL from the broadcasting network of $-100 \mathrm{dBm}$ for both scenarios (markers $2 \mathrm{c}$ and $4 \mathrm{c}$ in Figure 6). However, due to the strictest interference constraint, the spectrum usage increases 2.3 channels on average in the rural scenario (see markers $2 c$ and $2 \mathrm{a}$ in Figure 6) and 6.2 in the suburban scenario (see markers $4 \mathrm{c}$ and $4 \mathrm{a}$ in Figure 6). This considerably higher difference between both scenarios is due to a higher traffic density per square kilometer in the suburban region and a higher number of broadcasting services using different frequencies in the same region. This reduces the probability of reusing the same channels by the LTE-A BSs.

For an equal weight of both KPIs $\left(w_{1}=0.5 ; w_{2}=0.5\right)$ in the optimization algorithm, the interference from the broadcasting network compared with the best optimization case for the ISL KPI is increased by less than $0.2 \mathrm{~dB}$ (marker $2 \mathrm{~b}$ versus $2 \mathrm{c}$ in Figure 6) and $0.4 \mathrm{~dB}$ (marker $4 \mathrm{~b}$ versus $4 \mathrm{c}$ in Figure 6), for the rural and suburban scenarios, respectively. However, the spectrum usage is reduced by 1.5 and 2.7 channels, respectively (average for the whole area). Similar relative results are obtained for the LTE-A network self-interference (markers 1 and 3 in Figure 6).

We considered, for all cases, an equal weight when assessing the interference from the broadcasting network and the 
TABLE 2: Broadcasting signal settings for the experiments in the rural and suburban scenarios (configuration of the R\&S Broadcast Test Center).

\begin{tabular}{lccc}
\hline Parameter & $\begin{array}{c}\text { Rural } \\
\text { scenario }\end{array}$ & $\begin{array}{c}\text { Suburban } \\
\text { scenario }\end{array}$ & Unit \\
\hline Broadcasting standard & DTMB & DVB-T2 & $\mathrm{dBm}$ \\
Sensitivity threshold & -84.5 & -83.5 & $\mathrm{dBm}$ \\
Channel power & -83.5 & -82.5 & $\mathrm{dBm}$ \\
Modulation scheme & $64-$ & $64-\mathrm{QAM}$ & - \\
Coding scheme & $\mathrm{QAM}$ & $2 / 3$ & - \\
Interleaving & 0.6 & - & - \\
Frame header & 720 & - & - \\
Guard interval & $\mathrm{PN} 420$ & $1 / 16$ & - \\
OFDM subcarriers & $1 / 4$ & $32 \mathrm{k}$ & - \\
Pilot pattern & 3780 & PP2 & - \\
Maximum bitrate & - & 25.8718 & $\mathrm{Mbps}$ \\
Transport stream & 18.2738 & 23.6000 & $\mathrm{Mbps}$ \\
bitrate & 17.9837 & 91.2 & $\%$ \\
Used channel capacity & 98.4 & $1080 \mathrm{p}$ & - \\
Video resolution & $1080 \mathrm{p}$ & & \\
\hline
\end{tabular}

interference from the LTE-A devices, for the spectrum assignment in the LTE-A wireless network. For applications where the protection to/from a specific service has a higher relevance, a different ratio can be assigned for the optimization algorithm. The difference in absolute values is due to the different protection ranges for each technology (see Table 1 in Section 3.2).

Figure 7 shows the average interference reduction with respect to the maximum allowable interference for each scenario and weight combination at each simulation stamp. This is the GRAND algorithm optimization output. These datasets are transmitted to the FPGA for the experimental emulation (see Section 3.3.1).

In both scenarios, there is a variation between simulations depending on the users' distribution for a particular simulation. Depending on the user location relative to the BS and the EIRP, the interference will vary. The highest variation is $2 \mathrm{~dB}$ corresponding to the rural scenario. The standard deviation has an impact on the TOV and SFP emulation results. When the interference restriction becomes stricter (when $w_{2}=1$ ), the difference between simulations (different users' geolocations) is reduced: the connection decisions are based only on this KPI and the algorithm will lead to the lower limit of the interference threshold. This means the algorithm would likely connect more users at different frequencies for reducing the interference up to the minimum interference in the boundaries considered for the network optimization.

4.2. Experimental Assessment of the Interference to the Primary Licensed Service. Figure 8 shows the QoE metrics from the emulation measurement results as a function of the spectrum usage modelled by the optimization algorithm for the DTMB rural scenario and the DVB-T2 suburban scenario.

In our experiments, we do not compare the performance between DTMB and DVB-T2 but the performance of the optimization algorithm in two different scenarios. In terms of sensitivity, there is just $1 \mathrm{~dB}$ difference between the chosen configuration for DTMB and DVB-T2. The difference in the used data rate is lower than $7.2 \%$. Any difference in terms of interference is caused by the propagation conditions and broadcasting transmitters' radiation footprints, its relative geolocation, and the LTE-A traffic density per square kilometer in each scenario.

For the DTMB rural scenario (darker lines in Figure 8), the degradation of the perceived video quality at the edge of coverage of the broadcasting transmitter is similar for both metrics TOV (marker t\# in Figure 8) and SFP (marker s\# in Figure 8). The maximum recorded difference between both metrics for the rural scenario was $4.2 \%$ (see markers s1 and $\mathrm{t} 1$ in Figure 8). In the DVB-T2 suburban scenario, the maximum difference between SFP and TOV was $\sim 10 \%$ (see markers $t 6$ and $s 6$ in Figure 8). This was caused by more spaced burst errors. As the SFP stamps are shorter than the TOV, the number of timestamps affected is reduced. This effect is caused by a higher level of congestion in the rural LTE network under test with nearly constant load traffic higher than $95 \%$, compared with an average $80 \%$ to $90 \%$ (peak) in the suburban scenario.

For the optimization of spectrum usage without a dynamic range of the interference threshold and proper MCS management for minimizing interference, the percentage of timestamps with perceptible degradation is $65 \%$ and $69.2 \%$ in the rural measurements for the TOV and SFP indicators, respectively (markers $\mathrm{t} 1$ and $\mathrm{s} 1$ in Figure 8 ). In the suburban measurements, the TOV is exceeded on $62.5 \%$ of timestamps and $70 \%$ for the SFP indicator (markers $\mathrm{t} 5$ and s5 in Figure 8). The inclusion of interference optimization based on dynamic interference management (with a dynamic selection of the interference threshold in a caseby-case-based decision) has a significant impact on the video degradation minimization (as a QoE metric). For the worst optimization result, i.e., the software making decisions based on the spectrum result only $\left(w_{1}=1 ; w_{2}=0\right)$, the reduction in the video degradation is $50 \%$ to $58 \%$ in the rural measurements (see marker $\mathrm{t} 1$ versus $\mathrm{t} 2$ and marker s1 versus s2 in Figure 8 ) and $27.5 \%$ to $30 \%$ in the suburban region (see marker $\mathrm{t} 5$ versus $\mathrm{t} 6$ and marker s5 versus s6 in Figure 8 . The drawback is an $8 \%$ higher spectrum usage for both scenarios.

By assigning a certain channel, the LTE BS based on traditional cognitive radio technology will measure and assess the interference in a certain channel. For optimizing the spectrum usage, the BS will minimize the usage of channels, if the interference is below the defined interference threshold. This means the interference is not considered by the software as a KPI. For the channels of nearby transmitters, the mean interference will lead to the fixed interference threshold defined. With the introduction of interference as a KPI, even for the case of the highest weight for spectrum optimization, a lower interference is expected. This is because 


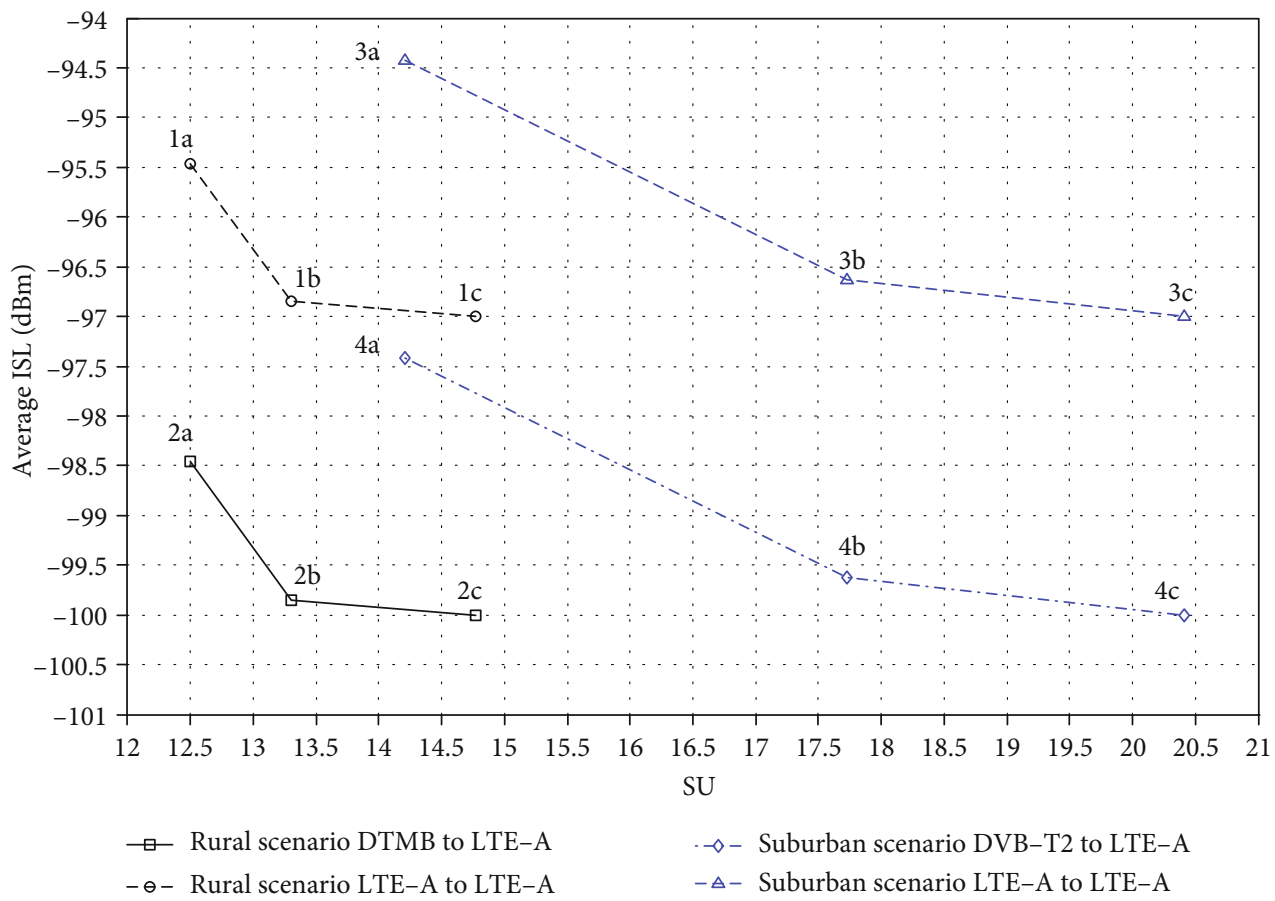

FIGURE 6: Optimized average ISL versus spectrum usage (SU: average number of channels used by the broadcasting primary service and the LTE-A secondary service), (a) corresponds to $w_{1}=1 ; w_{2}=0$, (b) $w_{1}=0.5 ; w_{2}=0.5$, and (c) $w_{1}=0 ; w_{2}=1$.

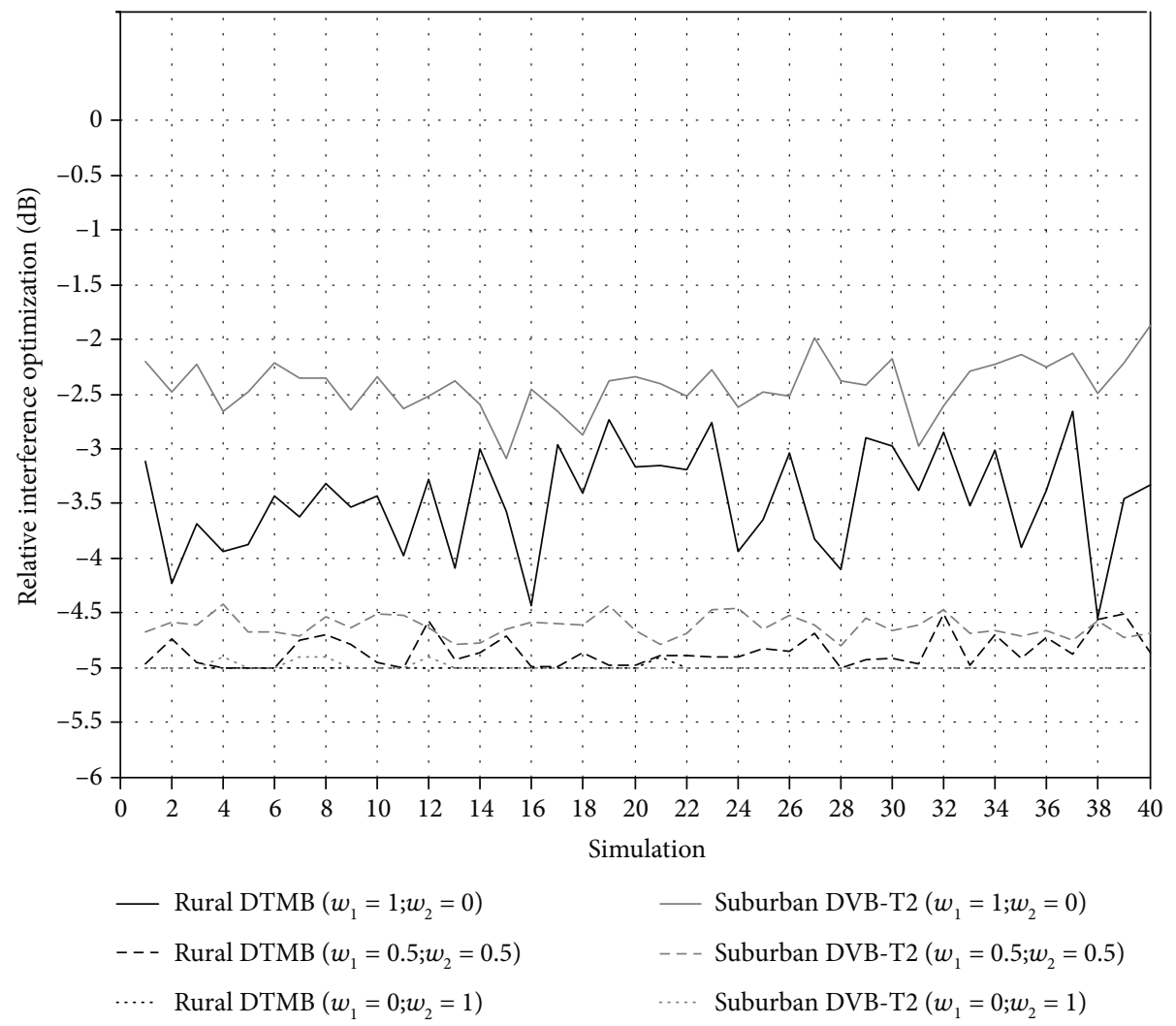

FIGURE 7: Algorithm outputs: relative interference with respect to the maximum allowable interference, i.e., minus optimization gain, $\left(-G_{O}\right)$, see Equation (5). These values are further used for setting the interference to the broadcasting coverage edge by the LTE-A network. 


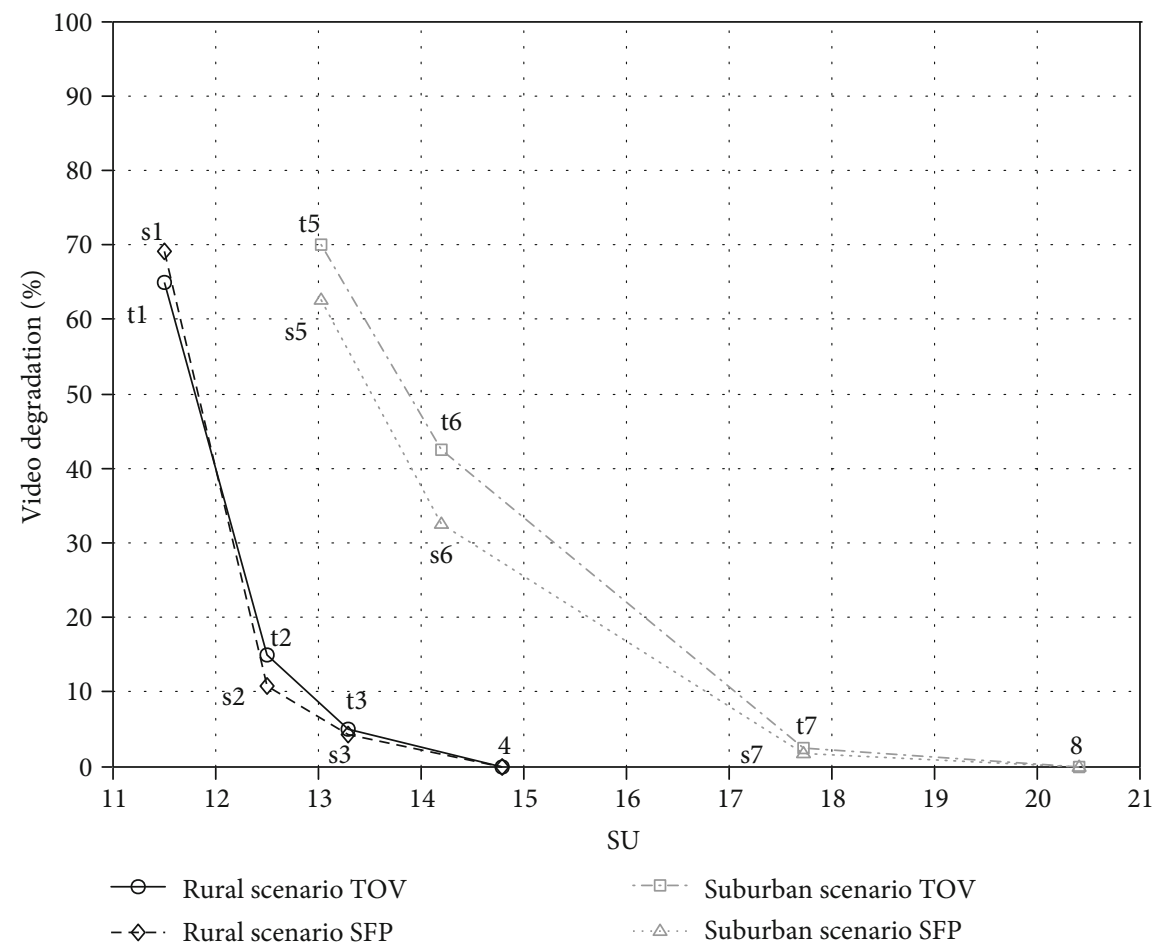

FIGURE 8: Video degradation versus average SU in the whole area. Marker s\# denotes TOV results, and markers t\# denote SFP results. Marker 1 represents the results for the traditional cognitive networks without dynamic interference management. For the dynamic interference management markers 2 represents the results for $G_{O}\left(w_{1}=1 ; w_{2}=0\right)$, marker 3 for $G_{O}\left(w_{1}=0.5 ; w_{2}=0.5\right)$, and marker 4 for $G_{O}\left(w_{1}=0\right.$; $\left.w_{2}=1\right)$.

a flexible range of interference allowance causes a higher degree of freedom for assigning the spectrum while keeping interference low. Hence, even for the case when the algorithm makes the user connection decisions based on the spectrum assignment results only, a slightly lower interference is achieved by the algorithm.

In the suburban scenario, the usage of frequencies by the primary broadcasting service is higher due to a higher density of traffic per square kilometer. As a consequence, the algorithm probability for reducing the interference is lower, taking into account that this KPI does not define the network optimization final result for the case of $w_{1}=1$ and $w_{2}=0$.

When the spectrum usage and interference metrics are equally weighted by the algorithm for deciding the user's connections, the SFP and TOV are exceeded in only $4.2 \%$ and $5 \%$ of the time for the rural measurements (markers $\mathrm{s} 3$ and $\mathrm{t} 3$ in Figure 8), and just $1.7 \%$ and $2.5 \%$ for the suburban measurements (markers s7 and $\mathrm{t} 7$ in Figure 8, SFP and TOV, respectively). As a drawback, spectrum usage is increased by $6 \%$ and $20 \%$ for the rural and suburban scenarios, respectively.

When interference minimization is the only KPI considered by the algorithm for deciding the user connections, there is no noticeable distortion in the transmitted video (markers 4 and 8 in Figure 8). Hence, in such a case, at the edge of the coverage of the broadcasting transmitter, there is no any disturbance in the users' QoE. The drawback is a higher spectrum usage by $10 \%$ compared with the one in marker s $3 \mid \mathrm{t} 3$ and $22 \%$ higher compared with marker $\mathrm{s} 1 \mid \mathrm{t} 1$ for the rural scenario. In the suburban scenario, the spectrum (marker 8 ) is increased by $13 \%$ compared with the one in marker s7|t7 and 36\% higher compared with marker s5|t5.

4.3. Experiment Result Interpretation. In a real LTE-A scenario based on the traditional distributed cognitive radio architecture, the protection margins recommended in the literature are not enough for protecting the primary broadcasting service from harmful interference. Our experiments revealed that for an LTE-A BS load higher than $80 \%$, the broadcasting video is degraded up to $70 \%$ of the time (at the broadcasting transmitter coverage edge). This is because such margins were developed for typical broadcasting scenario cases, not taking into account the variation of the transmitter's configurations and propagation environments for different scenarios. The variation in the broadcasting transmitters' height, MCS, EIRP, and environmental propagation conditions cause significant variability in the radiation footprints and the radius of the protection contour.

A simple reduction in the maximum allowable interference (e.g., from $-95 \mathrm{dBm}$ to $-97 \mathrm{dBm}$ ) might solve the interference problems, but at the cost of higher spectrum usage, that might be totally inefficient. For instance, reducing the maximum allowable interference threshold in the suburban area by $2 \mathrm{~dB}$, the spectrum usage increases by $17.1 \%$. Hence, a flexible interference range and dynamic interference management on a case-by-case basis must be applied, for guaranteeing a better trade-off between 
interference and spectrum usage. The designed algorithm for a centralized access management in the cognitive radio network allows reducing interference by at least $27 \%$ with just an $8 \%$ increase in spectrum usage.

\section{Conclusions}

In this paper, we presented an optimization algorithm for dynamic interference management in TVWS and for the minimization of spectrum occupancy. By emulating the critical conditions for the primary licensed service in an experimental testbed, we validated the algorithm performance for reducing harmful interference. Our experiments and measurements reveal that the protection margins proposed in the literature for DTMB and DVB-T2 cause severe interference when the LTE-A BS load is higher than $80 \%$. Because of the variability of the broadcasting station configurations, environmental and scenario characteristics, a flexible range for managing interference is more efficient and effective than defining a fixed constraint. The proposed algorithm for a centralized spectrum management architecture for cognitive radio technologies allows reducing the interference at least by $50 \%$ and $27.5 \%$ in the conducted rural and suburban subjective measurements, respectively, with just an $8 \%$ increase in spectrum usage.

Future work will consist of the implementation of our algorithm using a deep machine learning system for automated real-time interference and spectrum usage optimization.

\section{Data Availability}

Data underlying the findings of this study are openly available through Research Gate.

\section{Conflicts of Interest}

The authors declare that there is no conflict of interest regarding the publication of this paper.

\section{Acknowledgments}

This work has been supported in part by LACETEL, by the Special Research Fund BOF allocated to Ghent University by the Flemish Government, and by Rohde \& Schwarz. R. Martinez Alonso is supported by LACETEL and a doctoral grant from the Special Research Fund (BOF) of Ghent University, Belgium.

\section{References}

[1] L. Michael and D. Gómez-Barquero, "Bit-interleaved coded modulation (BICM) for ATSC 3.0," IEEE Transactions on Broadcasting, vol. 62, no. 1, pp. 181-188, 2016.

[2] L. Fay, L. Michael, D. Gómez-Barquero, N. Ammar, and M. W. Caldwell, "An overview of the ATSC 3.0 physical layer specification," IEEE Transactions on Broadcasting, vol. 62, no. 1, pp. 159-171, 2016.

[3] ETSI, ETSI EN 303 340: digital terrestrial TV broadcast receivers; harmonised standard covering the essential require- ments of article 3.2 of directive 2014/53/EU, ETSI, Cedex, France, 2016.

[4] FCC, FCC Auction 73: $700 \mathrm{MHz}$ Band, FCC, Washington DC, USA, 2008.

[5] Shared Spectrum Company, General survey of radio frequency bands - $30 \mathrm{MHz}$ to $3 \mathrm{GHz}$, Shared Spectrum Company, Vienna, Virginia, 2010.

[6] M. Hoyhtya, A. Mammela, M. Eskola et al., "Spectrum occupancy measurements: a survey and use of interference maps," IEEE Communications Surveys \& Tutorials, vol. 18, no. 4, pp. 2386-2414, 2016.

[7] J. van de Beek, J. Riihijarvi, and A. Achtzehn, "TV white space in Europe," IEEE Transactions on Mobile Computing, vol. 11, no. 2, pp. 178-188, 2011.

[8] A. Arteaga and A. Navarro, "Availability of TV white spaces using spectrum occupancy information and coverage maps," IEEE Latin America Transactions, vol. 14, no. 6, pp. 25882591, 2016.

[9] IEEE Computer Society, IEEE 802.11af. Part 11: wireless LAN medium access control (MAC) and physical layer (PHY) specifications. Amendment 5: television white spaces (TVWS) operation, IEEE, New York, US, 2013.

[10] IEEE Computer Society, IEEE 802.22b. Part 22: cognitive wireless RAN medium access control (MAC) and physical layer (PHY) specifications: policies and procedures for operation in the TV bands, IEEE, New York, 2015.

[11] ECMA, ECMA-392: MAC and PHY for operation in TV white space, ECMA International, Geneva, Switzerland, 2012.

[12] F. Granelli, P. Pawelczak, R. Prasad et al., "Standardization and research in cognitive and dynamic spectrum access networks: IEEE SCC41 efforts and other activities," IEEE Communications Magazine, vol. 48, no. 1, pp. 71-79, 2010.

[13] ETSI, TR 103067 reconfigurable radio systems (RRS); feasibility study on radio frequency $(R F)$ performance for cognitive radio systems operating in UHF TV band white spaces, ETSI, France, 2013.

[14] L. Montsi, L. Mfupe, F. Mekuria, and D. Macleod, "A real-time big-data system for smart spectrum sharing: enabling affordable broadband in $5 \mathrm{~g}$ for underserved communities," in Global Wireless Summit, Cape Town, South Africa, 2017.

[15] J. H. Martin, L. S. Dooley, and K. C. P. Wong, "5G multi-layer routing strategies for TV white space secondary user access," IET Communications, vol. 13, no. 12, pp. 1801-1807, 2019.

[16] ERICSSON, This is 5G, ERICSSON, Stockholm, Sweden, 2018.

[17] W. Prawatmuang, D. K. C. So, and E. Alsusa, "Sequential cooperative spectrum sensing technique in time varying channel," IEEE Transactions on Wireless Communications, vol. 13, no. 6, pp. 3394-3405, 2014.

[18] N. Tadayon and S. Aissa, "A multi-channel spectrum sensing fusion mechanism for cognitive radio networks: design and application to IEEE 802.22 WRANs," IEEE Transactions on Cognitive Communications and Networking, vol. 1, no. 4, pp. 359-371, 2015.

[19] A. Ebian, B. Abdelhamid, and S. El-Ramly, "New measurement matrix for compressive sensing in cognitive radio networks," IET Communications, vol. 12, no. 11, pp. 1297-1306, 2018.

[20] V. Popescu, M. Fadda, M. Murroni, J. Morgade, and P. Angueira, "Co-channel and adjacent channel interference and protection issues for DVB-T2 and IEEE 802.22 WRAN 
operation," IEEE Transactions on Broadcasting, vol. 60, no. 4, pp. 693-700, 2014.

[21] IEEE Computer Society, IEEE 802.22. Part 22: cognitive wireless RAN medium access control (MAC) and physical layer (PHY) specifications: policies and procedures for operation, IEEE, New York, 2011.

[22] FCC, FCC 19-24 Report, Federal Communications Commission, Washington DC, USA, 2019.

[23] A. Martínez Alonso, R. Martínez Alonso, and G. Guillén Nieto, "Parámetros de calidad de recepción del estándar DTMB en $6 \mathrm{MHz}$," in IEEE Biennial Congress of Argentina (ARGENCON), Bariloche, Argentina, 2014.

[24] V. Popescu, M. Fadda, and M. Murroni, "Performance analysis of IEEE 802.22 wireless regional area network in the presence of digital video broadcasting - second generation terrestrial broadcasting services," IET Communications, vol. 10, no. 8, pp. 922-928, 2016.

[25] D. Lekomtcev and R. Marsalek, "Comparison of 802.11af and 802.22 standards - physical layer and cognitive functionality," Electrorevue, vol. 3, no. 2, pp. 12-18, 2012.

[26] A. S. Cacciapuoti, M. Caleffi, and L. Paura, "Optimal strategy design for enabling the coexistence of Heterogeneous networks in TV white space," IEEE Transactions on Vehicular Technology, vol. 65, no. 9, pp. 7361-7373, 2016.

[27] S. Yuan, L. Li, and C. Chigan, "A selfishness-aware coexistence scheme for 802.22 and 802.11af networks," in IEEE Wireless Communications and Networking Conference Workshops, New Orleans, LA, USA, March 2015.

[28] R. Martinez Alonso, D. Plets, M. Deruyck, G. Guillen Nieto, L. Martens, and W. Joseph, "TV white space and LTE network optimization Toward energy efficiency in suburban and rural scenarios," IEEE Transactions on Broadcasting, vol. 64, no. 1, pp. 164-171, 2018.

[29] M. Zaeri-Amirani, F. Afghah, and S. Zeadally, "A hierarchical spectrum access scheme for TV white space coexistence in heterogeneous networks," IEEE Access, vol. 6, pp. 78992-79004, 2018.

[30] C. K. Huynh and W. C. Lee, "An efficient channel selection and power allocation scheme for TVWS based on interference analysis in smart metering infrastructure," Journal of Communications and Networks, vol. 18, no. 1, pp. 50-64, 2016.

[31] I. McGregor, "The relationship between simulation and emulation," in Proceedings of the Winter Simulation Conference, San Diego, CA, USA, December 2002.

[32] Standarization Administration of the PRC, GB 20600: framing structure, channel coding and modulation for digital television terrestrial broadcasting system (DTMB), Chinise National Standard, Beijing, China, 2006.

[33] ETSI, ETSI EN 302 755: digital video broadcasting (DVB); frame structure channel coding and modulation for a second generation digital terrestrial television broadcasting system (DVB-T2), EBU-ETSI, Cedex, France, 2012.

[34] ETSI, Physical layer aspects for evolved Universal Terrestrial Radio Access (UTRA), France, 2006.

[35] ETSI, TS 136104 release 12: LTE; Evolved Universal Terrestrial Radio Access (E-UTRA); base station (BS) radio transmission and reception, ETSI, Cedex, France, 2017.

[36] M. Deruyck, E. Tanghe, W. Joseph, and L. Martens, "Modelling and optimization of power consumption in wireless access networks," Computer Communications, vol. 34, no. 17, pp. 2036-2046, 2011.
[37] A. F. Molisch, Wireless Communications, John Wiley \& Sons, 2011.

[38] ITU-R, Recommendation ITU-R P.1546-5: method for pointto-area predictions for terrestrial services in the frequency range $30 \mathrm{MHz}$ to $3000 \mathrm{MHz}$, ITU, Geneva, 2013.

[39] Y. Oyarzábal Estopinan and A. Fernández Bezanilla, Generating digital television coverage map with MATLAB code, XXIII FORDES Technological Week, Havana, Cuba, 2013.

[40] D. Plets, W. Joseph, E. Tanghe, L. Verloock, and L. Martens, "Analysis of propagation of actual DVB-H signal in a suburban environment," IEEE Antennas-and-Propagation-Society International Symposium, vol. 1-12, pp. 1839-1842, 2007.

[41] M. Deruyck, J. Wyckmans, L. Martens, and W. Joseph, "Emergency ad-hoc networks by using drone mounted base stations for a disaster scenario," in 2016 IEEE 12th International Conference on Wireless and Mobile Computing, Networking and Communications (WiMob), pp. 1-7, New York, NY, USA, October 2016.

[42] ITU-R, Report ITU-R BT.2215-7: measurements of protection ratios and overload thresholds for broadcast $T V$ receivers, ITU-R, Geneva, Switzerland, 2018.

[43] ITU-R, Report BT.2035-2: guidelines and techniques for the evaluation of digital terrestrial television broadcasting systems including assessment of their coverage areas, ITU-R, Geneva, Switzerland, 2011.

[44] ITU-R, Recommendation BT.1368: planning criteria, including protection ratios, for digital terrestrial television services in the VHF/UHF bands, ITU-R, Geneva, Switzerland, 2017.

[45] P. Angueira, M. Velez, D. de la Vega, A. Arrinda, and J. L. Ordiales, "Fading caused by moving vehicles near the receiver on DTV (COFDM) 8-MHz signals," IEEE Communications Letters, vol. 6, no. 6, pp. 250-252, 2002. 